\title{
Speed of Synchrotron Radiation
}

\author{
VV Apollonov ${ }^{1 *}$ and Yu P Voinov ${ }^{2}$ \\ ${ }^{1}$ Prokhorov General Physics Institute of the Russian Academy of Sciences, Russia \\ ${ }^{2}$ Lebedev Physical Institute, Russian Academy of Sciences, Russia
}

*Corresponding author: VV Apollonov, Prokhorov General Physics Institute, Russian Academy of Sciences, Vavilova 38, 119991 Moscow, Russia

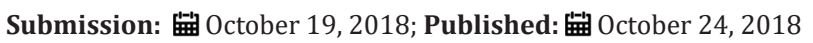

\author{
Abstract \\ A method for measuring and unambiguous interpretation of the results of measuring the speed of synchrotron radiation (speed of light) is \\ developed. \\ Keywords: Speed of light; Synchrotron; Special theory of relativity
}

\section{Introduction}

Papers [1,2] present two alternative approaches to the analysis of the measurement of the speed of synchrotron radiation (speed of light) on the Siberia-1 electron storage ring, with the results obtained indicating that the speed of light can be either $\mathrm{c}$ or $2 \mathrm{c}$. The dependence or independence of the speed of light on the source velocity is of fundamental importance for fundamental physics. The independence of the speed of light lies at the heart of the special theory of relativity and modern electrodynamics. Maxwell's equations of electrodynamics, which are invariant under the Lorentz transformations in any inertial system, show that the speed of light does not vary with the light source velocity. This conclusion affects many sections of physics--from optics to astrophysics. At one time there was a phlogiston theory (a fire-like element), which explained many thermal phenomena. Nevertheless, the phlogiston theory was overthrown by the kinetic theory of the motion of atoms and molecules that could adequately explain the nature of all thermodynamics.

At the same time, Maxwell's theory seems to anchor the ideological approach of the ether in the form of electromagnetic fields, the perturbation of which generates the propagation of electromagnetic waves. But the ether theory was refuted by the experiments of Michelson and Morley. Therefore, in our opinion, electrodynamics should be developed sooner or later that is adequate to the realm surrounding us and explains all optical phenomena and the nature of a photon, over which many prominent scientists (including academician O.N. Krokhin) still rack their brains.

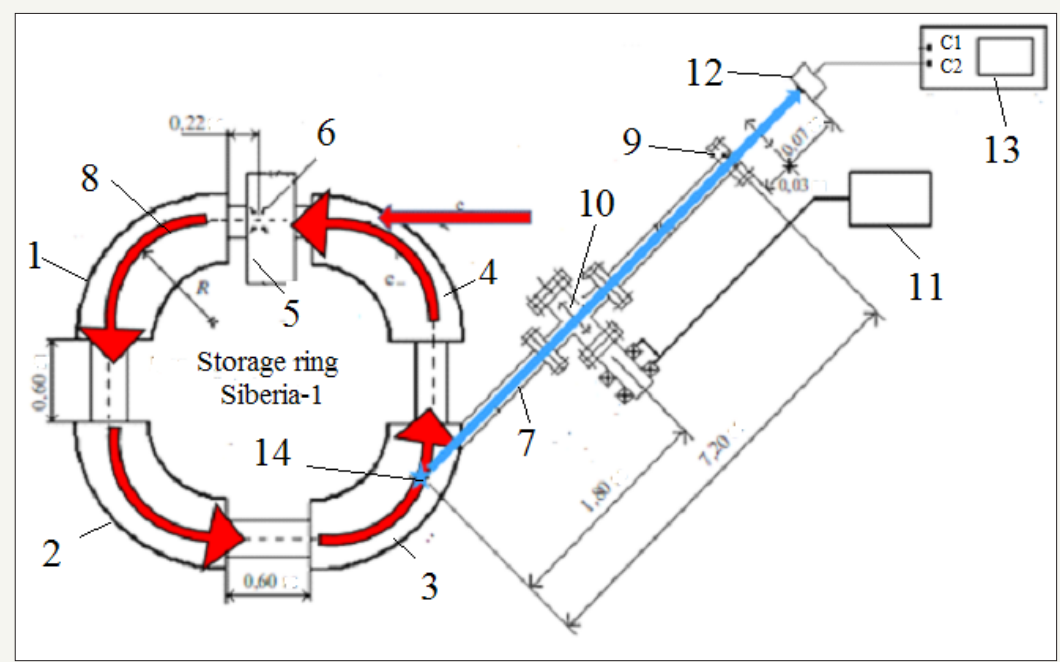

Figure 1: Schematic of the experiment: (1--4) rotary corner magnets; (5) resonator; (6) accelerating gap; (7) output channel of synchrotron radiation; (8) stationary orbit; (9) output window; (10) glass plate input/output system (IOS); (11) IOS control unit; (12) radiation detector; (13) oscilloscope; (14) point of emission. The distances are given in meters. 
In the light of what has been said, it is extremely important for us to carry out a control experiment that will unequivocally testify whether the synchrotron radiation velocity VSR obtained using the Sibiria-1 storage ring or its counterpart is equal to $2 \mathrm{c}$ or $\mathrm{c}$. The schematic of the experiment is shown in Figure 1. The red arrow indicates the path of the electrons, which produce synchrotron radiation (SR) in each curved section. At point of emission (14), the
SR is launched into the rectilinear SR output channel and measured with an optical detector (12). The information about the experiment and the source of synchrotron radiation--the Sibira-1 electron storage ring can be found in detail elsewhere $[1,2]$. To determine the speed of synchrotron radiation in the rectilinear segment of the output channel (7), use is made of the following measurement scheme (Figure 2).

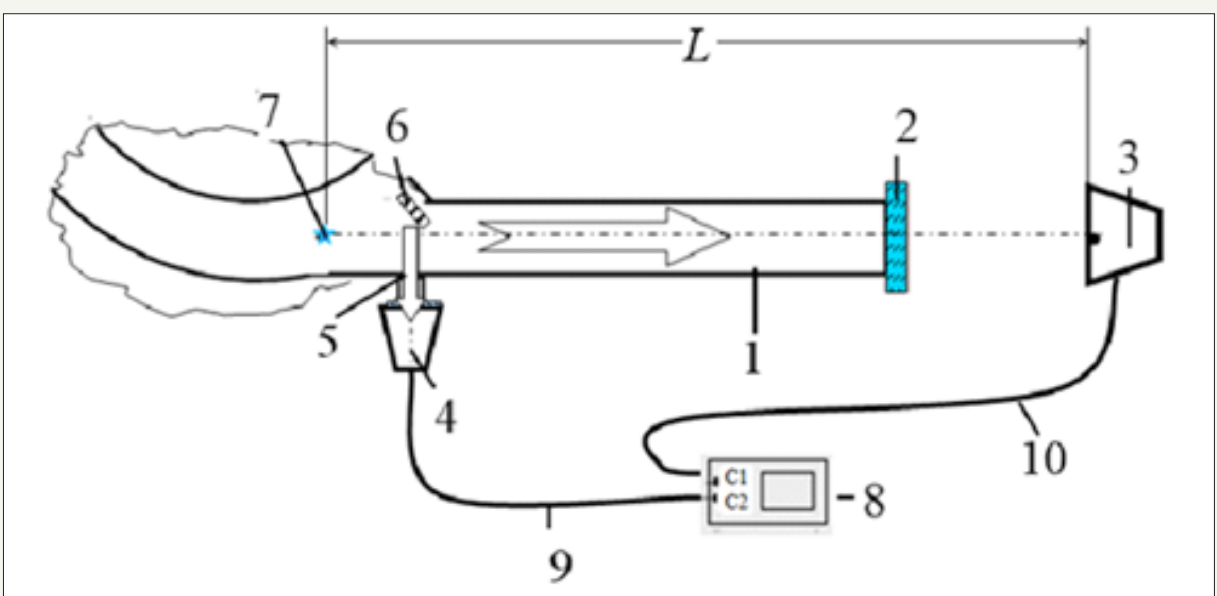

Figure 2: Proposed schematic of the measurements: (1) radiation output channel; (2) output window; (3) radiation detector of the measuring channel; (4) detector of the reference channel; (5) reference radiation output channel; (6) mirror of the reference channel output; (7) point of emission of an electron from a stationary orbit; (8) two-beam oscilloscope; (9) reference channel cable; (10) measuring channel cable.

Radiation detectors (3) and (4) must be pre-synchronized by changing the length of cables (9) and (10). To this end, the detectors are placed side by side and irradiated with a repetitively pulsed laser light, which makes it possible to synchronize signals in the measuring and reference channels of the oscilloscope. The measuring base L must satisfy the condition

\section{$L=(2 \mathrm{n}+1) \times T \times 30 \mathrm{~cm}$}

where $n=0,1,2,3, \ldots ;$ and $\mathrm{T}$ is the period of rotation of electrons along a circular orbit in the synchrotron (in ns).

When this condition is met, one of the following oscillograms will be observed on the oscilloscope screen (Figure 3).

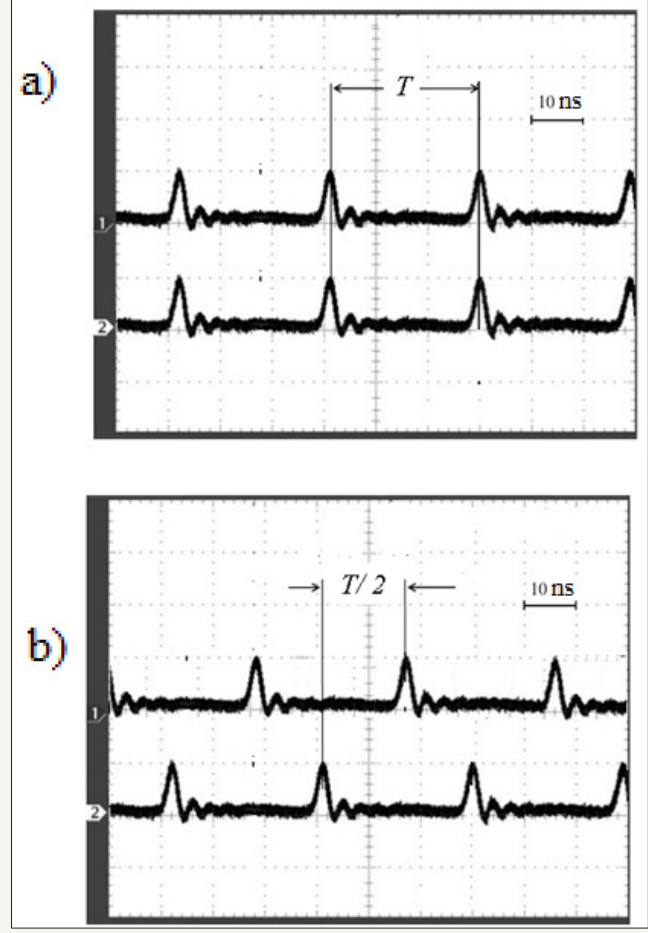

Figure 3: Oscillograms observed on the oscilloscope screen at a synchrotron radiation speed of light equal to (a) c and (b) $2 \mathrm{c}$. 
On the given oscillograms channel No. 1 registers a signal from the synchrotron radiation that has passed through the segment $\mathrm{L}$. Channel No. 2 serves as a reference channel recording the moments of light emission at point (7). For a qualitative picture, i.e. the answer to the question whether the speed of light is equal to $c$ or $2 c$, the segment from point (7) through mirror (6) to detector window (4) can be neglected as compared to the distance L (Figure 3). If it is necessary to accurately measure the speed of light of synchrotron radiation, then this segment should be considered.

For the Sibiria-1 electron storage ring, the minimum value of the segment, calculated from the above formula, is $\mathrm{L}=869 \mathrm{~cm}$. Therefore, for a real length $\mathrm{L} \sim 720 \mathrm{~cm}$, the shape of the oscillograms in Figure 3 for the two cases will slightly change. But all the same, one can immediately say that the speed is either c or 2c. If for technical reasons it is difficult to make use of the reference channel near point (14) in Figure 1, then it is possible to use a glass plate input/output system and measure the distance L from this place. Thus, we have proposed a simple experiment that will allow us to determine with high accuracy the dependence or independence of the speed of light on the light source velocity, which is of fundamental importance for fundamental physics. After all, this postulate is used to build not only the special theory of relativity, but also modern electrodynamics.

\section{References}

1. Aleksandrov EB, Aleksandrov PA, Zapasskii VS, Korchuganov VN, Stirin AI, et al. (2011) Direct experimental demonstration of the second special relativity postulate: the speed of light is independent of the speed of the source. Phys Usp 54(12): 1272.

2. Apollonov VV, Voinov YP (2017) Experiments directly demonstrating that the speed of light is independent of the velocity of the source: an experimental fact or a misconception. American Research Journal of Physics 3(1): 8
Creative Commons Attribution 4.0

International License

For possible submissions Click Here

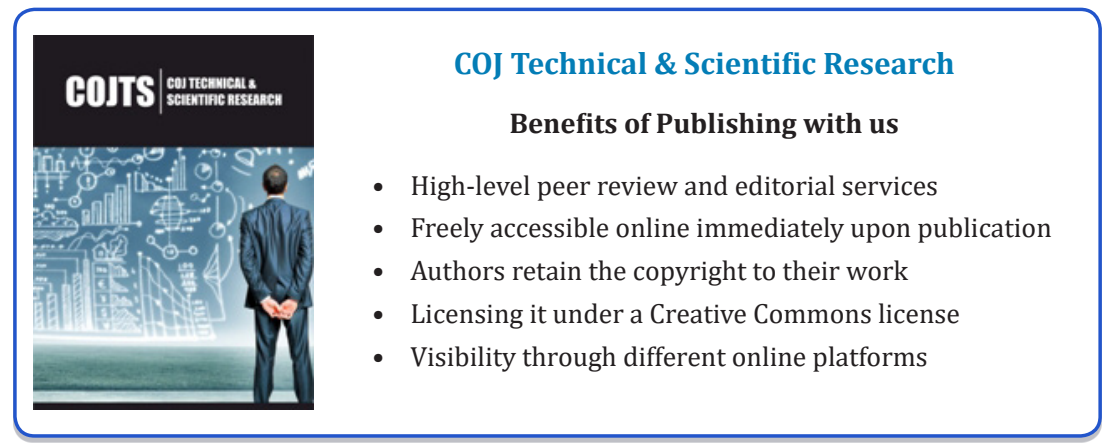

